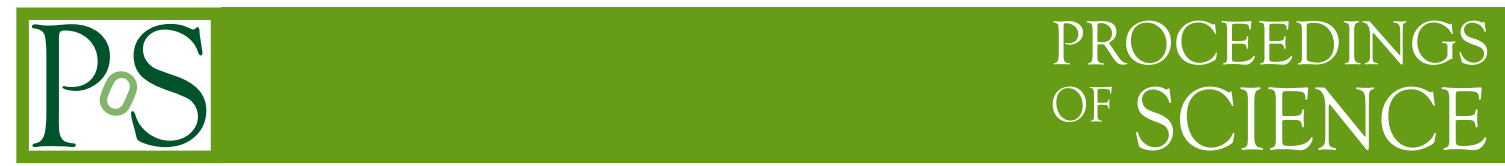

\title{
Weak Lensing: Status and Prospects
}

\author{
C. Danielle Leonard* \\ Carnegie Mellon University \\ E-mail: daniellleandrew.cmu.edu
}

Gravitational lensing is the effect in which light from distant sources is deflected by the intervening mass, resulting in distortions in observed images. Weak gravitational lensing, where this distortion is sufficiently small to be detectable only statistically, is a key cosmological observable, the measurement of which is a top priority of several current and next-generation cosmological galaxy surveys. With it, we can probe the evolution of dark energy, the nature of gravity, and other fundamental physical questions.

2nd World Summit: Exploring the Dark Side of the Universe 25-29 June, 2018 - EDSU2018

University of Antilles, Pointe-à-Pitre, Guadeloupe, France

${ }^{*}$ Speaker. 


\section{A Brief Review of Weak Lensing Theory}

In the weak regime, the gravitational lensing of galaxies is encapsulated by two effects [3]: convergence $\kappa$ (size and flux magnification) and shear $\gamma$ (distortion in galaxy shape). In practice, the shear is the standard observable of weak gravitational lensing surveys.

Two categories of galaxy weak lensing statistics are typically measured: cosmic shear and galaxy-galaxy lensing.

Cosmic shear is weak lensing of source galaxy images by the large-scale structure of the Universe. We measure two-point correlation functions between the shapes of source galaxy pairs, often considering auto- or cross-correlations between different tomographic redshift bins of source galaxies. The specific quantities of interest are the two-point correlation functions $\xi_{+}(\theta)$ and $\xi_{-}(\theta)$, given by (see, e.g., [22]):

$$
\xi_{ \pm}^{i, j}(\theta)=\frac{1}{2 \pi} \int_{0}^{\infty} C_{\ell}^{\kappa^{i} \kappa^{j}} J_{0 / 4}(\ell \theta) \ell d \ell
$$

where $\theta$ is angular separation, $C_{\ell}^{\kappa^{i} \kappa^{j}}$ is the power spectrum of the lensing convergence in tomographic bins $i$ and $j, l$ is the 2D on-sky Fourier mode conjugate to $\theta$ and $J_{i}$ are Bessel functions of the $i$ th order. The power spectrum of the convergence (in flat space and in the regime where the Limber approximation holds [16, 21]) is given by:

$$
C_{\ell}^{\kappa^{i} \kappa^{j}}(\ell)=\frac{9}{16} \int_{0}^{\chi_{\infty}} d \chi \frac{g_{i}(\chi) g_{j}(\chi)}{\chi^{2} c^{4}}(H(\chi) a(\chi))^{4} \Omega_{\mathrm{M}}(\chi)^{2} P_{\delta}\left(\frac{\ell}{\chi}, \chi\right),
$$

where $P_{\delta}$ is used to refer to the matter power spectrum. The lensing kernel $g$ is

$$
g_{i}(\chi)=2 \chi \int_{\chi}^{\chi_{\infty}} \frac{\chi^{\prime}-\chi}{\chi^{\prime}} N_{i}\left(\chi^{\prime}\right) d \chi^{\prime}
$$

and $N_{i}(\chi)$ is the distribution of source galaxies in comoving distance $\chi$ for redshift bin $i$.

Galaxy-galaxy lensing is the case in which, instead of measuring the two-point correlation of shear with shear, a correlation is measured between the shear of a population of background source galaxies, and the positions of another population of foreground lens galaxies. A key quantity in measurements of galaxy-galaxy lensing is the tangential shear, $\gamma_{\mathrm{t}}(\theta)$, the two point correlation function between source galaxy shapes and lens galaxy positions.

For comparison to equation 1.2 above, the Fourier-space cross-spectrum of the convergence with galaxy positions, which is qualitatively similar to the real-space $\gamma_{\mathrm{t}}(\theta)$, is given by (again in a flat Universe and under the Limber approximation):

$$
C_{\kappa^{i} g^{j}}^{\ell}=\frac{3}{4} \int_{0}^{\chi_{\infty}} d \chi b(\ell, \chi) \frac{g_{i}(\chi) W_{j}(\chi)}{\chi c^{2}}(H(\chi) a(\chi))^{2} \Omega_{\mathrm{M}}(\chi) P_{\delta}\left(\frac{\ell}{\chi}, \chi\right),
$$

where $b$ is the galaxy bias (the relationship between the lens galaxy and dark matter fields).

Together with galaxy clustering (the two-point correlation of galaxy positions) cosmic shear and galaxy-galaxy make up the components of the ' $3 \times 2$-point' analysis which has become a standard multiprobe analysis approach of late-time photometric surveys such as the Dark Energy Survey (DES, discussed further below, [6]). 


\section{Completed, Ongoing and Upcoming Weak Lensing Efforts}

Since the first detection of cosmic shear in 2000 [26], multiple surveys have measured weak gravitational lensing. For illustrative purposes, we now discuss the key features of several completed, ongoing, and upcoming surveys, without attempting to be exhaustive.

\subsection{Completed}

- The Canada-France-Hawaii Telescope Lensing Survey (CFHTLenS, [9]) used data collected between 2003 and 2009 on he Canada-France-Hawaii Telescope to make weak lensing measurements on 154 square degrees of sky down to a limiting magnitude of 24.5 in the $r$-band.

- The Sloan Digital Sky Survey I \& II (SDSS, [19]) used the Sloan Digital Survey Telescope between 2000 and 2008 to 10,000 square degrees of the sky. With this larger area than CFHTLenS, its limiting magnitude was fainter at 22 in the $r$-band.

- The Deep Lens Survey (DLS, [13]) used the Blanco and Mayall telescopes to image 18 square degrees of sky. The DLS survey area was small, but its imaging was deep, with limiting magnitude between 26 and 27.

\subsection{Ongoing}

- The Dark Energy Survey (DES), employs the Dark Energy Camera on the 4-meter Blanco telescope at the Cerra Tololo Inter-American Observatory, Chile. The DES wide-angle survey has an final observational area of 5000 square degrees over five years. Key cosmology results and data from a $3 \times 2$-point analysis with Year 1 of survey data (1300 square degrees) are public [1].

- The Kilo Degree Survey (KiDS) uses OmegaCAM on the VLT Survey Telescope located in the Atacama Dessert in Chile. The survey has a final observational area of 1500 square degrees. Cosmological results and data from the first 450 square degrees have been released $[25,14,10]$.

- Hyper-Suprime Cam (HSC) is a Japanese-led camera on the Subaru Telescope located at Mauna Kea in Hawaii. The collaboration has recently released the first weak lensing catalogues from the survey, using the first 137 square degrees [2]; the final survey area is anticipated to be 1400 square degrees. HSC is notable in that its limiting magnitude is comparable to that expected for LSST, making it an important precursor analysis data set.

\subsection{Upcoming}

- The Large Synoptic Survey Telescope (LSST, [18]) is an optical telescope currently under construction, with science goals of understanding dark energy, dark matter, and transient events. Located on the Cerro Pachón ridge in Chile, its principle wide survey aims to image 18,000 square degrees of the sky, with 20 billion galaxies anticipated to be observed in total over ten years from 2023.

- Euclid [7] is a satellite mission of the European Space Agency, planned for launch in 2021 with an expected lifetime of 6.5 years. It will survey 15,000 square degrees with the objective of providing images suitable for weak gravitational lensing, baryon acoustic oscillation, and redshiftspace distortion analysis. Euclid will measure weak lensing shapes for 1.5 billion galaxies.

- The Wide-Field Infrared Survey Telescope (WFIRST, [23]) is a US-led satellite mission with anticipated launch in the mid-2020s. It will image 2200 square degrees. With this smaller area than 
its contemporaries (LSST and Euclid), it will instead excel by focussing on considerably deeper imaging.

\section{Weak Lensing with LSST}

LSST will image more sky area, deeper, and for longer than any other ground-based optical survey. As such, there will be particular challenges to measuring weak lensing from LSST and to using these measurements to make inferences about cosmology [24]. These include:

- Shear estimation: Recent years have seen the development of creative techniques to measure shear without the need to rely on calibration from simulations, which could in principle fail to provide fully representative galaxy images. Metacalibration [12] is one such technique which was used in the DES Year 1 analysis [1]. Another, Bayesian Fourier Domain shear estimation, is under active development [4], and has been shown to by construction have subdominant multiplicative shear bias, an important source of systematic error.

- Photometric redshifts: To obtain redshift information for source galaxy samples, we rely on photometric redshifts. However, obtaining sufficiently well-calibrated redshift estimates is especially challenging for upcoming surveys. LSST will have a limiting magnitude much fainter than any previous optical wide-field imaging survey; it is not clear that it will be possible to obtain spectroscopic redshifts from a representative subsample of sources as has previously been possible. Photometric redshift estimation methods for LSST must be robust to this.

- Covariance estimation: Computing the multiprobe covariance is an extremely challenging feature of the analysis framework, with methodologies using many simulation realizations unlikely to scale well to LSST. Furthermore, standard assumptions must be re-examined in the context of the high statistical power of LSST. For example, efforts are ongoing to understand how the assumption that the likelihood of weak lensing is Gaussian may lead to parameter biases [20, 17].

- Intrinsic alignments: In addition to the correlations in shapes of galaxies due to weak lensing, galaxy shapes can be intrinsically correlated due to local tidal gravitational forces. This effect, known as intrinsic alignment, contaminates the weak lensing signal and if unmitigated, leads to biased inference and systematic errors. Several recent works have proposed or improved upon new methods to measure and / or mitigate intrinsic alignments in the LSST era, using self-calibration [27] and taking advantage of multiple shear estimation methods [15].

- Baryonic effects: At smaller cosmological scales, the $3 \times 2$-point observables are affected by baryonic physics such as, for example, feedback from Active Galactic Nuclei [5]. Methods are being developed to best mitigate the effect of the uncertain nature of baryonic physics on our results, including Principle Component Analysis methodologies [8, 11].

- Point-spread function: Effects from the atmosphere and telescope optics can cause spurious correlations in shapes of galaxy images. One way to minimize this is with the creative use of different methods of dithering exposures.

- De-blending: Galaxies which overlap on the sky (but are not necessarily close in redshift) cause significant issues for photometric redshift estimation and shape measurement for lensing. In current surveys the solution is often just to discard blended galaxies from the data set. However, with a deeper survey like LSST, we see more galaxies per area, and therefore more galaxies overlap; reliable de-blending methods will thus be essential for LSST science. 


\section{Conclusions}

Weak gravitational lensing will be one of the major observational tools which we will use to understand cosmology with next-generation surveys. To unlock the full power of weak lensing measurements from LSST and other upcoming surveys, there is considerable work to be done. This opportunity for the development of innovative techniques and methods has already led to exciting advances in the field.

\section{References}

[1] T. M. C. Abbott et al. Phys. Rev., D98(4):043526, 2018.

[2] H. Aihara et al. PASJ, 70:S8, January 2018.

[3] Matthias Bartelmann and Peter Schneider. Physics Reports, 340(4â̆ASS):291-472, 2001.

[4] G. M. Bernstein et al. MNRAS, 459:4467-4484, July 2016.

[5] N. E. Chisari et al. MNRAS, 480, 2018.

[6] Dark Energy Survey Collaboration et al. 2005.

[7] Euclid Collaboration. ESA/SRE, 12(1.1), 2011.

[8] T. Eifler et al. MNRAS, 454:2451-2471, December 2015.

[9] C. Heymans et al. MNRAS, 432:2433-2453, July 2013.

[10] H. Hildebrandt et al. MNRAS, 465:1454, 2017.

[11] H.-J. Huang et al. arXiv: 1809.01146, September 2018.

[12] E. Huff and R. Mandelbaum. arXiv: 1702.02600, February 2017.

[13] M. J. Jee et al. APJ, 765:74, March 2013.

[14] S. Joudaki et al. MNRAS, 474:4894-4924, March 2018.

[15] C. D. Leonard et al. MNRAS, 479, 2018.

[16] D. Nelson Limber. The Astrophysical Journal, 117, 1953.

[17] Chien-Hao Lin et al. In preparation.

[18] LSST Science Collaboration et al. arXiv: 0912.0201, December 2009.

[19] Rachel Mandelbaum et al. MNRAS, 432(2):1544-1575, 2013.

[20] E. Sellentin and A. F. Heavens. MNRAS, 473:2355-2363, January 2018.

[21] P. Simon. Astronomy \& Astrophysics, 473:711-714, October 2007.

[22] F. Simpson et al. MNRAS, 429(3), 2013.

[23] D. Spergel et al. ArXiv e-prints, March 2015.

[24] The LSST Dark Energy Science Collaboration et al. arXiv: 1809.01669, September 2018.

[25] Edo van Uitert et al. 2017.

[26] Ludovic Van Waerbeke et al. Astronomy and Astrophysics, 358:30-44, 2000.

[27] J. Yao et al. arXiv: 1809.07273, September 2018. 